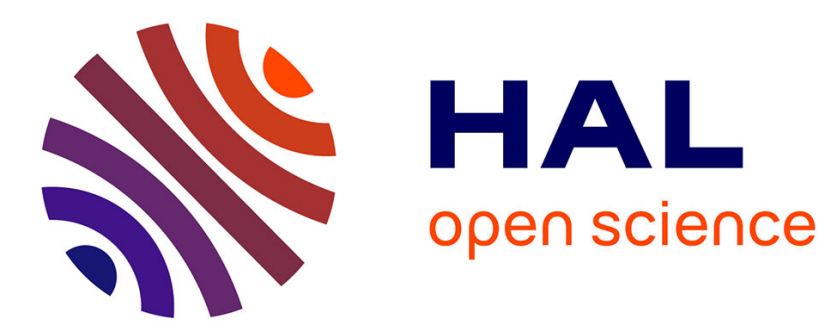

\title{
Régénération cardiaque : les leçons du développement Francesca Rochais
}

\section{To cite this version:}

Francesca Rochais. Régénération cardiaque : les leçons du développement. Archives des Maladies du Coeur et des Vaisseaux - Pratique, 2018, 2018 (271), pp.34-36. 10.1016/j.amcp.2018.09.001 . hal-02009367

\section{HAL Id: hal-02009367 https://hal-amu.archives-ouvertes.fr/hal-02009367}

Submitted on 6 Feb 2019

HAL is a multi-disciplinary open access archive for the deposit and dissemination of scientific research documents, whether they are published or not. The documents may come from teaching and research institutions in France or abroad, or from public or private research centers.
L'archive ouverte pluridisciplinaire HAL, est destinée au dépôt et à la diffusion de documents scientifiques de niveau recherche, publiés ou non, émanant des établissements d'enseignement et de recherche français ou étrangers, des laboratoires publics ou privés. 
REGENERATION CARDIAQUE : LES LEÇONS DU DEVELOPPEMENT

Francesca Rochais

Aix Marseille Université, INSERM, MMG U1251, 13005 Marseille, France.

E-mail : francesca.rochais@univ-amu.fr 
Les pathologies cardiovasculaires, incluant les défauts cardiaques congénitaux et les pathologies acquises, sont la cause majeure de mortalité dans les pays industrialisés (Writing Group et al., 2016). Les pathologies cardiovasculaires sont caractérisées initialement par des altérations moléculaires et cellulaires du myocarde, des artères coronaires ou des valves qui conduisent à une destruction massive des cardiomyocytes, associée à une altération de la fonction cardiaque résultant in fine en une insuffisance cardiaque chronique. Malgré de nombreuses avancées, les traitements conventionnels ne pallient pas le déficit en cardiomyocytes et le pronostic vital de l'insuffisance cardiaque reste encore insuffisant. Ainsi, le remplacement des cardiomyocytes détruits lors de la pathologie et la restauration d'une architecture cardiaque fonctionnelle constituent les cibles principales de la recherche médicale régénérative. Contrairement aux poissons zèbres adultes dont le cœur régénère totalement après blessure (Poss et al., 2002), le cœur de mammifère adulte ne régénère pas. Cependant de récentes études démontrant que le cœur de mammifère nouveau-né possède un potentiel de réparation endogène similaire à celui des poissons zèbres adultes (Porrello et al., 2011) suggèrent fortement qu'une compréhension détaillée des processus développementaux va non seulement permettre de comprendre l'étiologie des cardiopathies congénitales chez l'Homme mais est indispensable à l'identification de cibles thérapeutiques potentielles visant la réparation et la régénération cardiaque (Tzahor and Poss, 2017).

Le cœur est l'un des premiers organes à se former pendant l'embryogenèse. La cardiogenèse correspond à une séquence d'évènements permettant la transformation d'une structure simple, le tube cardiaque, en une structure complexe, le cœur définitif. Le développement du cœur peut être divisé en deux phases de croissance majeures (Figure 1). 
La première phase embryonnaire précoce repose sur la prolifération et l'ajout progressif au tube cardiaque en développement de nombreuses cellules progénitrices cardiaques. Lors de la seconde phase qui a lieu aux stades fœtaux, la croissance et le remodelage du cœur se produisent par la prolifération contrôlée des cardiomyocytes différentiés. La morphogenèse cardiaque commence, approximativement au jour embryonnaire (E) 7.5 chez la souris par la formation du croissant cardiaque. Rapidement (E8.0), les progéniteurs cardiaques du croissant cardiaque fusionnent au niveau de la ligne médiane pour donner naissance au tube cardiaque linéaire. Ce tube cardiaque, composé de deux couches cellulaires (une couche de cellules myocardiques qui entourent un tuyau de cellules endothéliales), possède deux extrémités : un pôle veineux situé postérieurement (la voie afférente) et un pôle artériel situé antérieurement (la voie efférente). A E8.5, une deuxième population de progéniteurs cardiaques provenant du mésoderme pharyngé et appelée second champ cardiaque (SHF pour Second Heart Field) va alors, par addition progressive de cellules au niveau des pôles artériel et veineux, participer à l'élongation rapide du tube cardiaque. La longueur du tube cardiaque embryonnaire va quadrupler entre E8 et E9.5. Situées dorsalement au tube cardiaque précoce, les cellules du SHF sont hautement prolifératives, expriment les gènes codant pour les facteurs de transcription $I s / 1$ et $T b \times 1$ et les facteurs de croissance $F g f 8$ et Fgf10 et se caractérisent par une différentiation retardée qui ne s'achève que lors de leur intégration au tube cardiaque (Kelly, 2012). L'allongement rapide du tube cardiaque, accompagné d'une inflexion vers la droite, conduit au positionnement correct des chambres cardiaques dans le cœur embryonnaire (E10.5 chez la souris ou quatrième semaine de développement chez l'homme). Plusieurs études de lignage génétique ont démontré que les cellules progénitrices du croissant cardiaque et du SHF contribuent à différents territoires du 
cœur adulte. En effet, alors que le croissant cardiaque participe à la formation du ventricule gauche et des oreillettes, les progéniteurs du SHF contribuent au ventricule droit, aux deux pôles du cœur et à une partie des oreillettes. D'autres types cellulaires, autres que les cellules progénitrices du myocarde, participent à la formation du cœur. Les cellules de la crête neurale cardiaque migrant du tube neural dorsal vers la voie efférente, participent directement à la formation des valves, à la séparation de l'aorte et du tronc pulmonaire et à la formation des structures dérivées des arcs pharyngés (gros vaisseaux) (Kelly, 2012). Plusieurs voies de signalisation issues de mécanismes cellules autonomes sont impliquées dans la régulation de l'addition progressive des progéniteurs du SHF aux pôles du cœur. Par ailleurs, les signaux extracellulaires dans la région pharyngée provenant des cellules de la crête neurale cardiaque, de l'ectoderme et de l'endoderme participent également à la régulation fine du déploiement des cellules du second champ cardiaque. De nombreuses études démontrent que des altérations de ces voies de signalisation affectent le déploiement des cellules progénitrices du second champ cardiaque et conduisent in fine à des défauts cardiaques congénitaux (Rochais et al., 2009).

A partir du dixième jour de développement (chez la souris) ce n'est plus l'ajout de cellules progénitrices qui permet la croissance des cavités cardiaques mais la prolifération contrôlée des cardiomyocytes différentiés. Une régulation spatio-temporelle précise de la prolifération des cardiomyocytes fœtaux est nécessaire à la formation correcte du cœur et toute altération de cette régulation fine conduira à des malformations cardiaques congénitales (Ahuja et al., 2007). En plus des mécanismes cellulaires autonomes (Rochais et al., 2014), des signaux paracrines provenant de l'endocarde, de l'épicarde et des fibroblastes cardiaques ont été impliqués (Smith and Bader, 2007). Le potentiel prolifératif des 
cardiomyocytes diminue considérablement après la première semaine de vie (chez la souris). La croissance post-natale du cœur se poursuivra alors non plus par division cellulaire mais par augmentation du volume cellulaire des cardiomyocytes (croissance hypertrophique). Les cellules du proépicarde, situées sous le cœur embryonnaire à E9.5, vont migrer et recouvrir le cœur afin de former l'épicarde. Les cellules de l'épicarde vont à leur tour envahir le myocarde pour donner naissance aux fibroblastes et aux cellules musculaires lisses présentes au niveau des artères coronaires. Les cellules endothéliales coronaires, quant à elles, se différencient à partir des cellules de l'endocarde et du sinus veineux (Tian et al., 2015). Parallèlement à ces processus, entre E10.5 et E13.5, le cœur embryonnaire est soumis à une série de remodelages qui aboutit à la séparation de la circulation systémique et pulmonaire.

L'enrichissement considérable, ces dernières années, de nos connaissances du développement du cœur incluant la découverte des cellules progénitrices cardiaques embryonnaires précoces, l'identification des programmes transcriptionnels précis permettant la spécification des différents lignages cardiaques mais également la description des mécanismes moléculaires contrôlant la prolifération des cardiomyocytes fœtaux ont alors permis de réorienter et de guider les stratégies thérapeutiques régénératives les plus prometteuses à ce jour à savoir l'utilisation de progéniteurs cardiaques et l'activation de la prolifération de cardiomyocyte préexistants (Figure 1).

La découverte de progéniteurs cardiaques résiduels dans le cœur adulte est l'un des sujets le plus controversé depuis ces vingt dernières années dans le domaine de la régénération cardiaque. Plusieurs types de progéniteurs cardiaques résidents (CPC pour cardiac progenitor cells) ont été décrits dans le cœur adulte. Extrêmement rares et ils se 
caractérisent par l'expression de différents marqueurs membranaires (cKit, Sca1 ou PDGFR $\alpha$ ) ou facteur de transcription (Is|1). Les CPC-cKit ${ }^{+}$ont été les plus étudiés et rapidement des capacités régénératives remarquables leur ont été attribuées (Nadal-Ginard et al., 2014). Cependant, de récentes études analysant précisément l'identité, l'origine développementale et la capacité des CPC à se différentier en cardiomyocytes, ont permis de révéler que les capacités régénératives des CPC semblent en fait extrêmement limitées. Les effets bénéfiques observés après leur transplantation semblent être principalement dus à des effets paracrines angiogéniques et pro-survie (Cai and Molkentin, 2017).

L'utilisation de cellules souches embryonnaires ou pluripotentes induites ou encore la reprogrammation directe de cellules cardiaques non-musculaires représentent l'application concrète de nos connaissances précises des facteurs de transcription et voies de signalisation embryonnaires qui gouvernent la spécification et la différentiation des progéniteurs cardiaques au cours du développement. En effet, les protocoles précis de reprogrammation et de différenciation in vitro utilisant les mécanismes moléculaires initialement décrits comme ayant un rôle clé dans les étapes de spécification des lignages cardiaques embryonnaires permettent aujourd'hui de générer des populations de cardiomyocytes relativement pures et conséquentes; prérequis indispensables pour leur injection directe dans le myocarde ou leur intégration dans des matrices biologiques afin de générer des patchs réparateurs contractiles (Tzahor and Poss, 2017).

Aujourd'hui il apparait clairement que la perte des capacités prolifératives des cardiomyocytes après la naissance soit à l'origine de la perte des capacités régénératives cardiaques des mammifères. Contrairement aux considérations antérieures, le cœur adulte de mammifère, y compris chez l'Homme, n'est pas un organe post-mitotique. En effet, un 
renouvellement ininterrompu de cardiomyocytes au cours de la vie (entre 0.5 et $1 \%$ par an chez un Homme adulte) a été décrit et les nouveaux cardiomyocytes générés sont issus de cardiomyocytes préexistants et non pas de progéniteurs résiduels (Senyo et al., 2013). Ainsi, bien que limité, l'existence d'un remplacement continuel des cardiomyocytes supporte le développement de stratégies thérapeutiques régénératives visant à stimuler ce processus. Cette stratégie très prometteuse nécessite cependant une compréhension détaillée des mécanismes moléculaires qui contrôlent la prolifération des cardiomyocytes au cours du développement fœetal et qui conduisent à leur sortie du cycle cellulaire après la naissance (Galdos et al., 2017). Récemment plusieurs voies de signalisation comme la voie Hippo (Yap/TEAD), Neuréguline (Nrg1/ErbB2), FGF ou encore les niveaux de ROS intracellulaires suscitent un intérêt majeur et semblent représenter des cibles thérapeutiques extrêmement intéressantes pour les stratégies réparatrices et régénératives cardiaques (Tzahor and Poss, 2017).

Ainsi, l'ensemble de ces évidences démontre à quel point nos connaissances du développement du cœur ont participé au progrès rapide de la médecine expérimentale régénérative. Ces dernières découvertes et les efforts en cours vont incontestablement conduire à de futures avancées indispensables pour le remplacement des cardiomyocytes détruits lors des pathologies cardiovasculaires congénitales et acquises et ainsi favoriser la restauration d'une architecture cardiaque fonctionnelle. 


\section{Références}

Ahuja, P., et al., 2007. Physiol Rev 87, 521-544.

Cai, C.L., Molkentin, J.D., 2017. Circ Res 120, 400-406.

Galdos, F.X., et al., 2017. Circ Res 120, 941-959.

Kelly, R.G., 2012. Curr Top Dev Biol 100, 33-65.

Nadal-Ginard, B., et al., 2014. Stem Cell Res 13, 615-630.

Porrello, E.R., et al., 2011. Science 331, 1078-1080.

Poss, K.D., et al., 2002. Science 298, 2188-2190.

Rochais, F., et al., 2009. Circ Res 104, 933-942.

Rochais, F., et al., 2014. Cardiovasc Res 104, 432-442.

Senyo, S.E., et al., 2013. Nature 493, 433-436.

Smith, T.K., Bader, D.M., 2007. Semin Cell Dev Biol 18, 84-89.

Tian, X., et al., 2015. Circ Res 116, 515-530.

Tzahor, E., Poss, K.D., 2017. Science 356, 1035-1039.

Writing Group, M., et al., 2016. Circulation 133, e38-360.

Figure 1: Stratégies thérapeutiques régénératives cardiaques: apport des connaissances du développement du cœur.

Schéma représentant les deux phases majeures du développement du cœur chez la souris. Les cellules progénitrices du second champ cardiaque et les territoires cardiaques dérivés sont représentés en bleu. L'identification des mécanismes moléculaires contrôlant le destin des progéniteurs cardiaques et la prolifération des cardiomyocytes au cours du développement contribue fortement aux stratégies thérapeutiques régénératives qui sont d'une part la stimulation/injection de progéniteurs cardiaques et d'autre part l'activation de la prolifération des cardiomyocytes préexistants. Aorte (Ao), cellules souches embryonnaires (ESC) et pluripotentes induites (iPSC), oreillette droite (OD), oreillette gauche (OG), tronc pulmonaire (Tp), tube cardiaque (TC), ventricule droit (VD) et ventricule gauche (VG). 


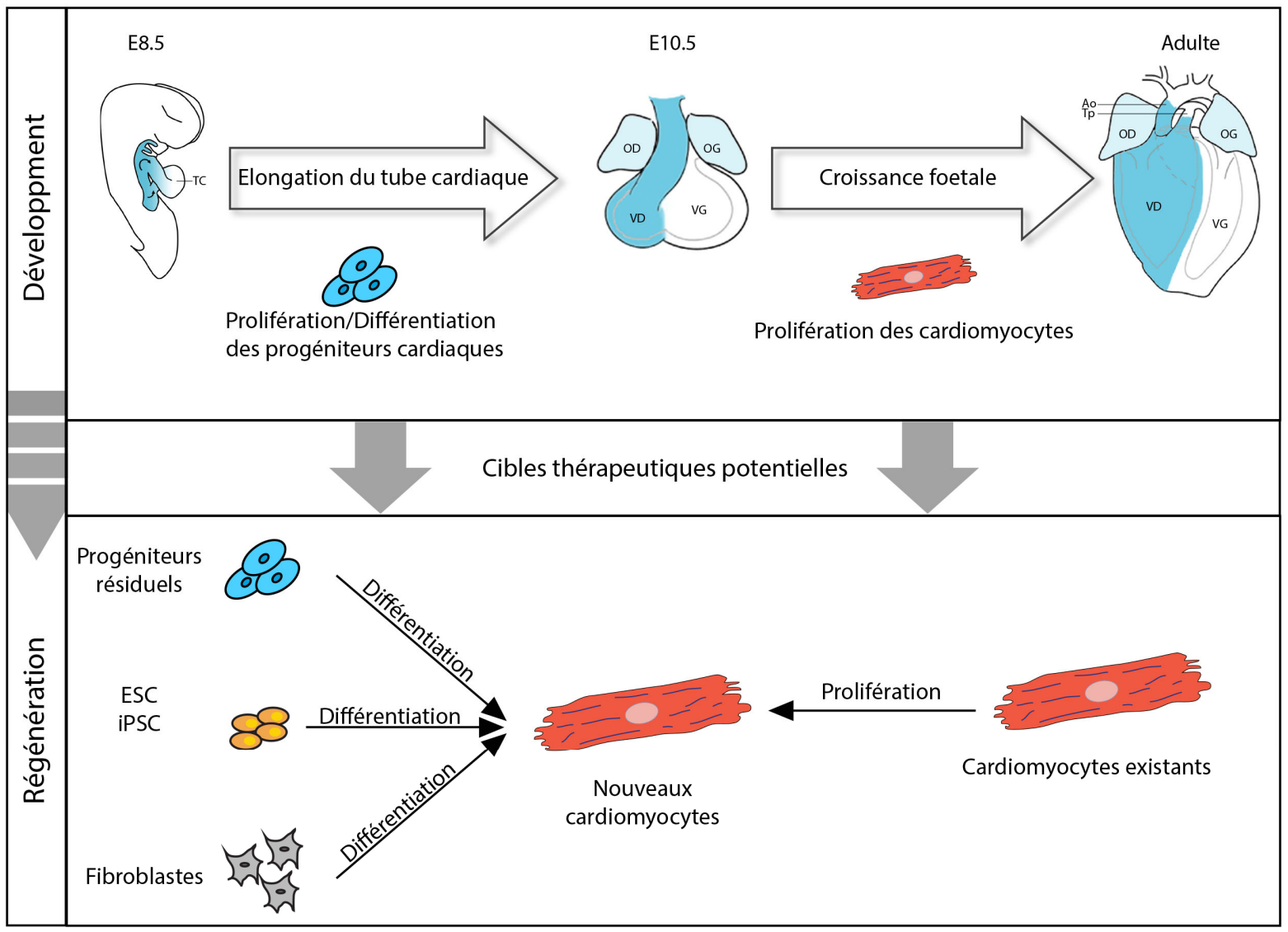

